\title{
Tres pinturas valencianas inéditas de Joanes, Requena y Espinosa
}

\author{
Albert Ferrer Orts \\ Universidad Autónoma de Chile ${ }^{1}$
}

\section{Beatriu Navarro Buenaventura, Josep Lluís Cebrián i Molina, Estefania Ferrer del Río}

Universitat de València

El objetivo de este breve estudio es dar a conocer tres pinturas pertenecientes a obradores valencianos de los siglos XVI y XVII que, dispersas y en el mercado del arte, muestran su relación con la órbita de Joan de Joanes, Gaspar Requena y Jerónimo Jacinto de Espinosa.

\section{Santa Ana, la Virgen y el Niño}

Atribuible a Joan de Joanes (c. 1505/10-1579)2 , es una tabla (óleo, $57 \times 44 \mathrm{~cm}$ ) prácticamente desconocida que recientemente ha formado parte de la exposición de los fondos del Ayuntamiento de Alcoi (Alicante) durante la primavera de 2013 [1]. La obra, cuyo origen se desconoce, fue adquirida por el consistorio en $1947^{3}$ y presenta, en primer término, a María sentada con el Niño en su regazo señalando el libro que sostiene abierto Santa Ana, en el que originalmente se debió leer: DICO VOBIS QUE /... UNQUE PETI / PATREM /... E MEO 4 , es decir, parte del discurso de despedida de Jesús después de la Última Cena. Los personajes se recortan sobre un paisaje montañoso con arquitecturas de reminiscencia clásica desdibujadas por la lejanía, mientras que la composición es idéntica a la de la espina del retablo desmembrado de San Sebastián, San Bruno y San Vicente Ferrer, procedente de la cartuja de Valldecrist en Altura (Castellón), c. 15405. Se aprecia una leve diferencia en la mano de la Virgen que, en idéntica posición, sostiene una flor en la tabla alcoyana y el paño de pureza del Niño en la pintura castellonense. La disposición de los detalles laterales de la escena también cambia, pues aparecen de manera invertida las arquitecturas del fondo y la masa arbórea, esta última con la adición de una oropéndola posada, que hemos visto en otras obras de Joanes como el Bautismo

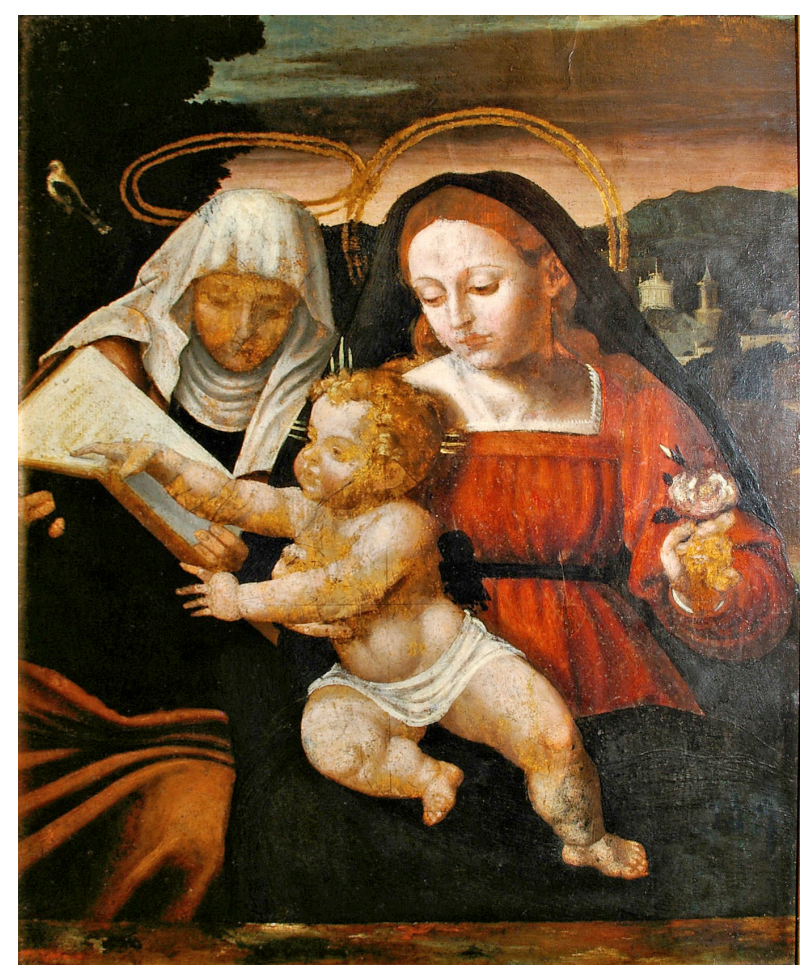

1. Joan de Joanes, Santa Ana, la Virgen y el Niño, Ayuntamiento de Alcoi, fotografía de J.L.C.M.

de Cristo, c. 1535, de la catedral de Valencia. Otras concordancias que no pasan desapercibidas la vinculan igualmente con el Tríptico de la Encarnación que el pintor realizó para las dominicas de Xàtiva (Valencia), c. 1550.

Los modelos iconográficos en los que se basa esta obra fueron señalados por Fernando Benito al estudiar la pieza de Altura, además de advertir la corpulencia piombes- 


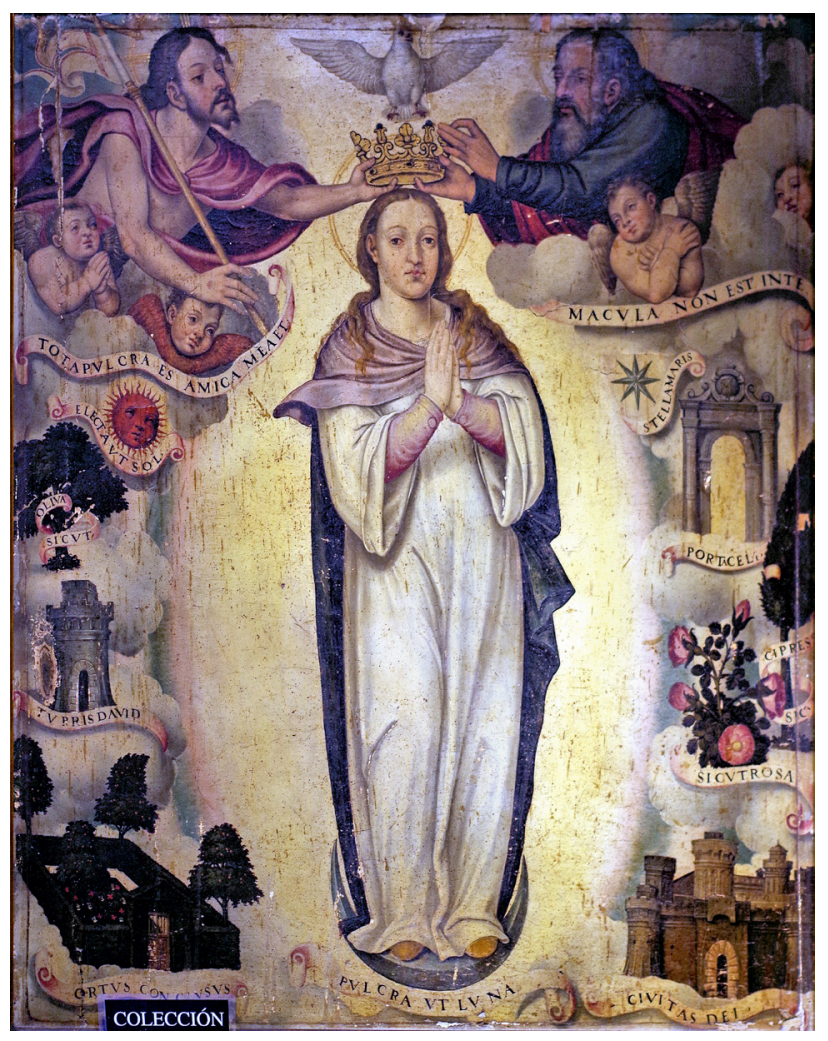

2. Gaspar Requena, Inmaculada, en el comercio, Valencia, fotografía de J.L.C.M.

ca de las figuras 6 . Santa Ana con toca y gesto concentrado se inspira en la Anna Triplex de Yáñez de la Almedina, que Joanes pudo ver en la parroquia de San Nicolás de Valencia; tipología que, por otra parte, ya había utilizado Vicente Macip en la tabla del mismo tema del Museo de Bellas Artes de Valencia. El modelo mariano, de elaboración propia, es el mismo que vemos en la Virgen del Loreto de la colección Perdigó de Barcelona 7 , y la postura del Niño es de ascendencia leonardesca a partir de la Virgen de los Husos.

La pintura ha sido recortada por la parte derecha, como puede apreciarse por la composición, ahora un tanto descentrada, y en el libro incompleto que sostiene Santa Ana. En conjunto está bastante arrasada ya que, al parecer, fue objeto de una restauración poco afortunada, pues fue «limpiada» de forma agresiva con disolventes que eliminaron la pintura en zonas muy amplias, como se puede ver en el rostro de Santa Ana o el cuerpo de Jesús, llegando incluso a disgregar parte de la preparación o, en el mejor de los casos, a hacer desaparecer las veladuras tanto en el rostro de María como en su ropaje. En algunas zonas el daño fue tan grave que ha dejado al descubierto el dibujo preliminar y parte de la cuadrícula. A parte del dibujo propio, que vemos en el rostro de Jesús, aparecen otras líneas que nada tienen que ver con el tema representado, lo que indicaría que es una tabla reaprovechada. Las aureolas, bastante toscas, son un repinte, pues seguramente las originales fueron destruidas en la misma actuación. En 1992 fue restaurada de nuevo, eliminando los añadidos y suciedad acumulada, así como consolidando la delicada capa pictórica ${ }^{8}$.

\section{Coronación de la Inmaculada Concepción}

Esta tabla (óleo, 100x80 cm aprox.) [2], en el comercio de arte valenciano, es obra innegable de Gaspar Requena (c. 1515-después de 1585 $)^{9}$, quien colaboró con Joanes en la realización del retablo mayor de la iglesia de la Font de la Figuera (Valencia) a mediados del siglo XVI y, muy probablemente, con antelación a este como denotan algunas obras que en su día reseñamos ${ }^{10}$. No cabe duda de su cercanía al maestro cuando en otras pinturas reproduce fielmente composiciones pertenecientes al acervo artístico de Joanes, como así lo manifiestan, a modo de ejemplo, la Trinidad que corona el conjunto de la parroquial de Montesa (1559), ligeramente posterior al retablo de San Antonio, Santa Bárbara y los Santos Médicos de Onda; o esta que presentamos, inspirada especialmente en la Inmaculada de Vicente Macip y Joanes, 1537 (antigua Colección Banco Central-Hispano, Madrid), y en la archiconocida Inmaculada del templo de la Compañía de Jesús (Valencia), c. 1570, de la que se conocen diversas versiones. No es esta la única ocasión en que Requena se sirve del exitoso modelo de Joanes para sus Inmaculadas, pensemos por ejemplo en el retablo homónimo de la iglesia de la Mercé de Xàtiva, en el retablito de San Antonio y la Inmaculada del Museo de la Catedral de Valencia o en la aureola pintada de los padres escolapios de Gandia ${ }^{11}$.

Sin embargo, el estilo de Requena, siendo fiel al del maestro e inspirador, dista de su excelencia, aunque en algunas ocasiones ha propiciado su confusión con obras de amplia participación del taller de Joanes. Su dominio de la anatomía humana es un tanto retardataria, siendo muy pe- 
culiares las fisonomías de sus personajes algo desproporcionadas y menos naturalistas, dotados de grandes cuencas oculares, ojeras, narices ligeramente redondeadas y aguileñas o rostros sonrosados, generosos e inanes, detalles que les confieren cierta falta de expresión y movilidad hasta privarles de la sutil comunicación íntima y ductilidad joanesca. Con todo, Requena se muestra como un buen colorista. De hecho colaboró estrechamente con Joanes, aunque, como en la obra que presentamos, utilice el pan de oro como fondo de la Purísima Concepción, muy en la línea de la tabla que en su día formó parte del retablo mayor de la desaparecida parroquia de San Bartolomé (Valencia).

\section{Santo Tomás dando limosna}

Óleo sobre lienzo $(165 \times 137 \mathrm{~cm})^{12}$ localizado en el altar dedicado al santo ${ }^{13}$, que también lo fue por un tiempo de Ntra. Sra. del Sagrado Corazón mientras la pintura estuvo en el Museo Colonial de San Francisco, en la actual iglesia de Nuestra Señora de Gracia (más conocida como de San Agustín) de Santiago de Chile, antaño templo del convento de agustinos de dicha ciudad, concretamente en el lado de la Epístola, y que ha sido atribuido tanto a Joanes -sin fundamento alguno- como a Zurbarán ${ }^{14}$ [3]. Se trata de una versión más de la escena con la que comúnmente se representa al que fuera monje agustino y arzobispo de Valencia (beatificado en 1618 y canonizado en 1658) desde que Joanes realizara el retrato de su efigie post mortem y, años más tarde (después de 1568), se basara en él para representarlo en la galería de mitrados que se halla en el aula capitular de la catedral valenciana ${ }^{15}$. Obra $(83 \times 56 \mathrm{~cm})$, en paradero desconocido, en la que se inspiraron a posteriori pintores tan reputados como Francisco de Zurbarán (1598-1664), Jerónimo Jacinto de Espinosa (1600-1667), Bartolomé Esteban Murillo (1617-1682), Mateo Cerezo (1626-1666) o Luca Giordano (1634-1705).

De la versiones conocidas que hemos podido ver de dichos artífices, atendiendo a su estilo, composición y paleta cromática, nos inclinamos a pensar que la obra en cuestión se acerca más al pincel del valenciano Espinosa, autor del que se conoce una versión del santo agustino fundamentada precisamente en la efigie que inmortalizara Joanes ${ }^{16}$, ya que también ejecutó por voluntad del cabildo valentino

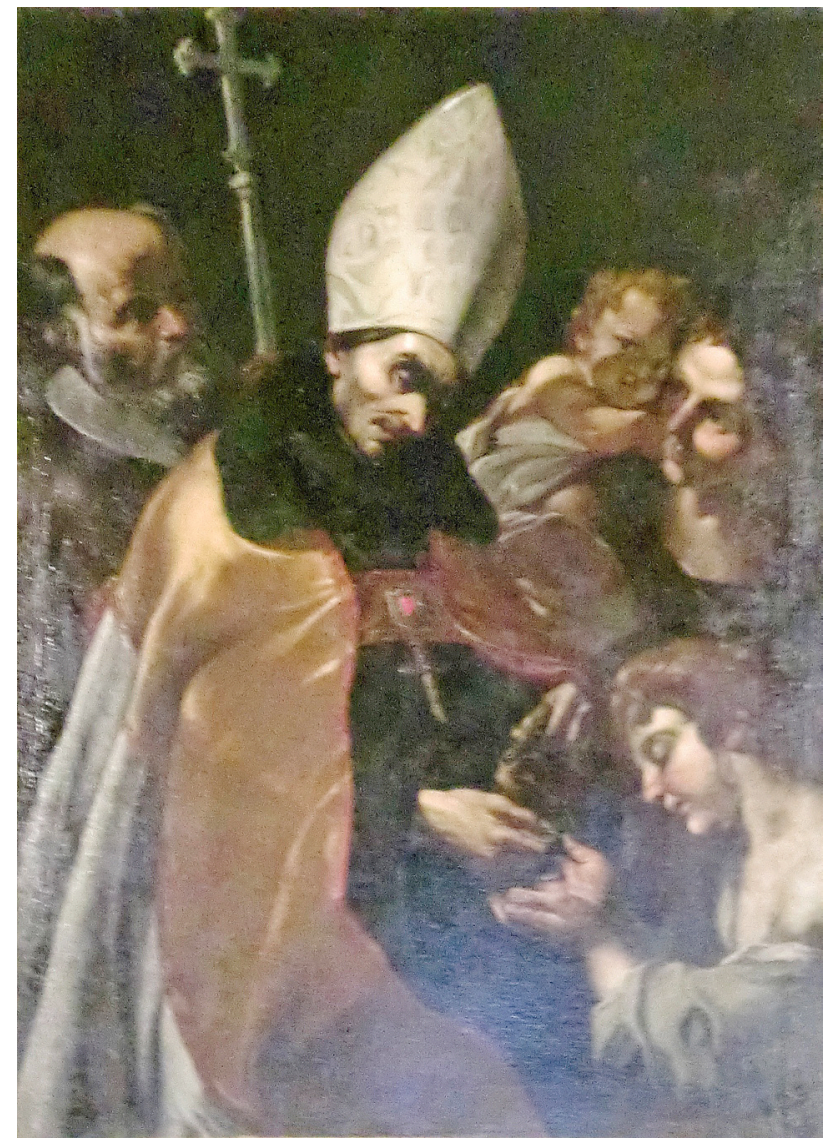

3. J. J. de Espinosa, Santo Tomás dando limosna, iglesia de Nuestra Señora de Gracia, Santiago de Chile, fotografía de A.F.O.

los retratos de algunos arzobispos, quizás los de Pedro de Urbina y Martín López de Ontiveros.

Nuestra hipótesis se basa en primer lugar en su origen, su intensa relación artística tanto con la sede metropolitana como con su diócesis, donde pintó para sus parroquias y para distintas congregaciones religiosas; y en su inconfundible estilo, estudiado en profundidad por Alfonso E. Pérez Sánchez. La obra chilena, muy barnizada y sin firmar, tiene resabios que la conectan con la pintura que se conserva en el Museo de Bellas Artes de Valencia $(108 \times 85 \mathrm{~cm})^{17}$, y, por ende, con la de Joanes, pues se trata de un rostro inspirado en la vera effigies del difunto. El santo aparece vestido de pontifical tornasolado con mitra junto a un clérigo que sostiene la cruz episcopal a sus espaldas, una mujer piadosa acercando a su hijo al santo y un adolescente pobre con el torso descubierto recibiendo la limosna en actitud de acción 
de gracias. Todo envuelto en un aura tenebrista tan afín a su estilo y, con él, a la pintura barroca española del período.

Queda por dilucidar cómo llegó esta obra a América, así como su ubicación final en la iglesia de los agustinos de Santiago de Chile, un templo que fue levantado hasta en tres ocasiones y que también sirvió de academia militar entre 1817 y el ecuador de la centuria; aunque en la biografía de Espinosa hay ciertas lagunas cronológicas entre 1640 y 1646 que sugieren la idea de que pudo viajar a Orihuela, Murcia, Madrid y Sevilla, lugar este último desde el que quizá realizara algunas obras destinadas al Nuevo Mundo ${ }^{18}$, tal como hiciera su contemporáneo Zurbarán y otros colegas. De lo que no cabe duda es que Espinosa viajó a tierras toledanas, ya que de su estancia manchega ha quedado obra autógrafa ${ }^{19}$.

\section{Notas}

1 Miembro del proyecto de investigación I+R+D del Ministerio de Economía y Competitividad, Plan Nacional: La consolidación de la pintura del Renacimiento en la Corona de Aragón: la extraordinaria influencia del paradigma de Joan de Joanes (HAR2012-32199); investigador principal X. Company, Universitat de Lleida.

2 Es indudable su sesgo joanesco, pero su pésimo estado de conservación nos impide certificar si hubo o no participación de taller.

3 SEGURA MARTÍ, Josep Maria, SANTAMARÍA CUELLO, Miquel, Colllecció d'Art de l'Ajuntament d'Alcoi. I Catàleg de pintures. Del segle XVI a la dècada de 1930, Alcoi, Ajuntament d'Alcoi, 2013, p. 12. En el Libro de registro de entrada del Museo de Arte consta su adquisición por parte del consistorio alcoyano el 20 de octubre de 1947. Referencia actual de la tabla: P-113.

$4 \mathrm{Jn}, 14: 12-14$

5 Las tablas dispersas del retablo de la cartuja de Altura se encuentran en diferentes colecciones y museos. El ático, con Santa Ana, la Virgen y el Niño, en colección particular de Madrid; la tabla central con San Sebastián y la Piedad de la predela en el Museo de Bellas Artes de Valencia; y las tablas laterales de San Bruno y San Vicente Ferrer en el MNAC. Recuérdese, a este propósito, que el conjunto fue expuesto en fechas recientes (2009-2010) en el museo valenciano.

6 BENITO DOMÉNECH, Fernando, GALDÓN, José Luis, Vicente Macip (1475-1550), Valencia, Generalitat, 1997, p. 148.

7 La tabla de la colección Perdigó es idéntica a la del convento de Santa Clara de Xàtiva, también atribuible a Joanes aunque muy repintada, pero que no ha sido tomada en consideración en la mayor parte de estudios dedicados al pintor desde que F. Benito la diera como desaparecida. BENITO, Fernando (com.), Joan de Joanes. Una nueva visión del artista y su obra, Valencia, Generalitat, 2000, p. 225. En el año 2001, cuando la comunidad religiosa, muy mermada, abandonó el convento y se trasladó a la vecina localidad de Canals, se llevó consigo los bienes muebles histórico-artísticos.

8 BARROS MONTERO, Ángel, BARROS GARCÍA, J. Manuel, Informe previo y memoria de restauración: Colección de pinturas del Ayuntamiento de Alcoy. Febrero-octubre de 1992, pp. 18-27. Queremos dejar constancia de nuestro agradecimiento a Josep Maria Segura, director del Museu Arqueològic Camil Visedo d'Alcoi, siempre tan dispuesto a la investigación de sus fondos.

9 HERNÁNDEZ GUARDIOLA, Lorenzo, et al., Gaspar Requena (c. 1515-después 1585). Pintor valenciano del Renacimiento, en prensa. A la hora de escribir estas líneas, la tabla se halla en el comercio de antigüedades Ars Antiqua (Valencia). Agradecemos a su propietario la posibilidad de realizar fotografías de la obra.

10 PÉREZ BURCHES, Inmaculada, «Aportación documental al retablo mayor de la iglesia de la Natividad de la Font de la Figuera de Joan de Joanes", Ars Longa, 17, 2008, pp. 25-33. FERRER ORTS, Albert; AGUILAR DÍAZ, Carmen, «Influjos joanescos en el taller de los Requena", Boletín del Museo e Instituto Camón Aznar, n. ${ }^{\circ}$ 104, 2009, pp. 127-145.

11 CEBRIÁN I MOLINA, Josep Lluís, «Difusió del patrimoni històric i artístic (l)», Papers de la Costera, 7-8, 1992, pp. 177-188. FERRER ORTS, Albert; AGUILAR DÍAZ, Carmen, "Més obres de Gaspar Requena el Jove i el seu actiu taller a la Costera", Ars Longa, 17, 2008, pp. 35-37. FERRER ORTS, Albert, AGUILAR DÍAZ, Carmen, «Noves obres atribuïbles a Gaspar Requena el Jove (ca. 1530-ca. 1603) i el seu taller», Boletín de la Sociedad Castellonense de Cultura, LXXXV, 2009, pp. 424-431. NAVARRO I BUENAVENTURA, Beatriu; CEBRIÁN I MOLINA, Josep Lluís, "Noves atribucions al pintor Gaspar Requena”, Revista de la Safor, 5, 2014, pp. 67-80.

12 Dimensiones con el marco incluido. El cuadro, situado en su altar a una altura considerable, está taladrado a la pared. Las medidas del lienzo sin el mismo son de $133^{\prime} 5 \times 96 \mathrm{~cm}$. Manifestamos desde estas líneas nuestro sincero agradecimiento a D. Guillermo Carrasco Notario, bibliotecario de los agustinos en Santiago, quien allanó las dificultades para realizar nuestro trabajo en el templo.

13 VILLAREJO, P. A. (OSA), Iglesia de San Agustín. Monumento Histórico-Nacional. Síntesis de vida santiaguina y agustiniana durante 400 años, Santiago, Ed. Agustinianas, 1994, p. 63, relata que el altar existía a finales del siglo XVIII. Es curioso constatar como un lienzo de San Agustín que fue enviado a los agustinos de México por Santo Tomás de Villanueva en 1535, y que de allí pasó a Lima, se custodió en el convento de Santiago desde 1595 hasta su desaparición en 1647 (Ibid., pp. 18-19).

14 ARAVENA, Héctor, Exposición de pintura clásica española, Santiago de Chile, Instituto Chileno de Cultura Hispánica, 1952, p. 34 y lámina XV; VILLAREJO, Iglesia de San Agustín..., pp. 89-90. La reproducción que aparece corresponde al negativo, por lo que la escena se muestra en sentido inverso a su correcta lectura. Su autoría no se basa en documentación alguna ni se aporta cómo pudo llegar al entonces convento. Circunstancia que tampoco aclaran ni MATURANA, P. Víctor (OSA), Historia de los agustinos de Chile, Santiago, 1904, ni CARRASCO NOTARIO, Guillermo, Luces y sombras. 400 años de presencia agustina en Chile: 1585-1995, Santiago, Ed. Agustinianas, 1995. Sospechamos que, de recalar en el siglo XVII, sería después del sismo del 13 de mayo de 1647, movimiento telúrico que asoló el templo y del que -como relatan los PP. Gaspar de Villarroel y Juan de Toro- únicamente se libró de la destrucción la escultura del Señor de la Agonía o de Mayo, obra del P. Pedro de Figueroa (c. 1612). Datos que, pensamos, pueden reforzar la idea de que Espinosa pudo estar en Sevilla y, desde allí, entrar en contacto con los canales del comercio artístico trasatlántico.

15 GARíN, Felipe V., Joan de Joanes (+1579), Valencia, Ministerio de Cultura, 1979, pp. 146-147.

16 PÉREZ SÁNCHEZ, Alfonso E., Jacinto Jerónimo de Espinosa (1600-1667), Valencia, Generalitat, 2000, pp. 168-169.

17 Ibidem.

18 Ibid., pp. 33-36. TORRALBA MESAS, Desirée, «Una obra del pintor Jeronimo Jacinto de Espinosa: La Santísima Trinidad de la Iglesia de Santiago de Santa Cruz de la Zarza", Archivo de Arte Valenciano, LXXXIX, 2008, pp. 53-63.

19 Ibidem. 